\title{
Análisis de asociación de los polimorfismos -725C>G (rs1233334), -201G>A (rs1233333) y 14 bp deleción/ inserción (14-pb del/ins) (rs66554220) del gen HLA-G en mujeres mexicanas con pérdida gestacional recurrente
}

\author{
Angela Porras D.1,3,a, Alma Benita Lazcano $C^{2}$, Thiago Donizete Da Silva J.1,3,b, \\ Clara Ibet Juárez V. ${ }^{3,4}$, Jesús Alejandro Juárez O. 1,3,c, Francisco Javier Perea D. 1,3,d, \\ José Elías García 0.1 \\ 1 División de Genética, Centro de Investigación Biomédica de Occidente, Instituto Mexicano del Seguro Social, Gua- \\ dalajara. 2 Servicio de Ginecoobstetricia, Hospital General 180, Instituto Mexicano del Seguro Social, Tlajomulco de \\ Zuñiga. ${ }^{3}$ Doctorado en Genética Humana, Centro Universitario de Ciencias de la Salud, Universidad de Guadalajara, \\ Guadalajara. ${ }^{4}$ División de Medicina Molecular, Centro de Investigación Biomédica de Occidente, Instituto Mexicano del \\ Seguro Social, Guadalajara. Jalisco, México
}

${ }^{\text {a }}$ Químico Farmacéutico, Biólogo; ${ }^{b}$ Biomédico; ${ }^{c}$ Biotecnólogo; ${ }^{d}$ Biólogo. Programa de Doctorado en Genética Humana. Universidad de Guadalajara, México.

\section{RESUMEN}

Antecedentes: El antígeno leucocitario humano (HLA)-G es una molécula inmunomoduladora que contribuye a la aceptación del feto semialogénico. Algunos polimorfismos de un solo nucleótido (SNP) en las regiones no codificantes del gen HLA-G inducen a la disminución de moléculas HLA-G, lo cual contribuye a complicaciones en el embarazo, tales como la preeclampsia o pérdida gestacional recurrente. Objetivo: Analizar la asociación de los polimorfismos -725C>G (rs1233334), -201G>A (rs1233333) y 14 bp deleción/ inserción (14-pb del/ins) (rs66554220) del gen HLA-G en mujeres mexicanas con PGR. Métodos: Los polimorfismos -725C>G ( $r$ 1233334), -201G $>A$ ( $r$ 1233333) y 14-pb del/ins ( $r$ 66554220) se identificaron por medio de PCR-SSOP (Polymerase Chain Reaction-sequence-specific oligonucleotide probe) y PCR (Polymerase Chain Reaction), respectivamente, en 58 mujeres con pérdida gestacional recurrente ( $\geq 2$ abortos), sin factores de riesgo identificables y 56 mujeres fértiles no relacionadas ( $\geq 2$ nacidos vivos). Resultados: El polimorfismo -725C>G (rs1233334) presentó diferencias significativas entre los grupos de estudio pero no se asoció con PGR ( $p=0,02601$; OR=11,484; IC95\%=0,617-213,659). Los polimorfismos $-201 \mathrm{G}>\mathrm{A}$ ( $\mathrm{rs} 1233333$ ) y 14-pb del/ins (rs66554220) no se distribuyeron de manera diferente entre los grupos de estudio ni se asociaron con pérdida gestacional recurrente. Los polimorfismos analizados se encontraron en equilibrio de ligamiento ( $D^{\prime}>0,3563 ; r^{2}<0,1140$ ). Conclusión. Este estudio sugiere que los polimorfismos -725C>G (rs1233334), -201G>A (rs1233333) y 14-pb del/ins (rs66554220) del gen HLA-G están en equilibrio de ligamiento y no influyen en el riesgo de pérdida gestacional recurrente en mujeres mexicanas.

PALABRAS CLAVE: Antígeno leucocitario humano, pérdida gestacional recurrente 


\section{SUMMARY}

Background: The human leukocyte antigen (HLA)-G is an important immunomodulatory molecule that contributes to the acceptance of the semi-allogeneic fetus. Some single nucleotide polymorphisms (SNP) in the noncoding regions of the HLA-G gene may influence the cellular levels of HLA-G, contributing to pregnancy complications such as preeclampsia or recurrent pregnancy loss. Objective: To analyze the association of $-725 \mathrm{C}>\mathrm{G}$ ( $\mathrm{rs} 1233334),-201 \mathrm{G}>\mathrm{A}$ ( $\mathrm{rs} 1233333)$ and $14 \mathrm{bp}$ deletion/insertion (14-bp del/ins) (rs66554220) polymorphisms in the HLA-G gene in Mexican women with RPL. Methods: -725C>G (rs1233334), -201G>A (rs1233333) and 14-bp del/ins (rs66554220) polymorphisms in the HLA-G gene were identified by PCRSSOP (polymerase chain reaction-sequence-specific oligonucleotide probe) and PCR (polymerase chain reaction), respectively, in 58 women with recurrent pregnancy loss ( $\geq 2$ miscarriages) without identifiable risk factors and 56 unrelated fertile women ( $\geq 2$ live births). Results: $-725 C>G$ (rs1233334) polymorphism showed significant differences between the study groups but it was not associated with recurrent pregnancy loss ( $\mathrm{p}=0.02601, \mathrm{OR}=11.484 ; 95 \% \mathrm{Cl}=0.617-213.659)$. $-201 \mathrm{G}>\mathrm{A}$ ( $\mathrm{rs} 1233333)$ and $14-\mathrm{bp}$ del/ins (rs66554220) polymorphisms were not distributed differently in study groups and not associated with RPL. Analyzed polymorphisms were in linkage disequilibrium ( $\left.D^{\prime}>0.3563, r^{2}<0.1140\right)$. Conclusion: This study suggests that $-725 \mathrm{C}>\mathrm{G}$ (rs1233334), $-201 \mathrm{G}>\mathrm{A}$ (rs1233333) and 14-pb del/ins (rs66554220) in the HLA-G gene are in linkage equilibrium and do not influence the risk of recurrent pregnancy loss in Mexican women.

\section{KEY WORDS: Human leukocyte antigen, recurrent pregnancy loss}

\section{INTRODUCCIÓN}

Pérdida gestacional recurrente (PGR) se define como dos o más pérdidas consecutivas antes de la semana 20 del embarazo, afecta aproximadamente al $5 \%$ de las mujeres en edad fértil, tiene etiología multifactorial y presenta un $50 \%$ de causas desconocidas (1-4). El gen HLA-G se encuentra en $6 \mathrm{p} 21.3$, se expresan preferentemente en el citotrofoblasto y tiene propiedades inmunosupresoras que contribuyen a que la madre acepte al feto semialogénico (5-8).

Algunos polimorfismos de un solo nucleótido (SNP) en las regiones no codificadoras del gen HLA$G$ se han asociado con PGR, por ejemplo: $-725 C>G$ (rs1233334) y 14 pb deleción/inserción (14-pb del/ ins) (ATTTGTTCATGCCT) (rs66554220) (9-11). El polimorfismo -725C>G (rs1233334) se localiza 19 pb río abajo del elemento ISRE, el cual une a IRF1 , en tanto durante la transcripción, la presencia del alelo $-725 \mathrm{G}$ contribuye a metilación e interfiere en dicho proceso $(8,12)$. El polimorfismo $-201 \mathrm{G}>\mathrm{A}$ (rs1233333) se localiza cerca del elemento RREB1 , el cual regula la baja transcripción del gen HLAG (8). El polimorfismo 14-pb del/ins (rs66554220) se encuentra en la posición 3741 de la región 3' no traducida del gen HLA-G. La presencia del alelo 14$\mathrm{pb}$ ins provoca una deleción de $92 \mathrm{pb}$ que influye en la estabilidad del ARNm y causa menor producción de HLA-G (13-15). Por tanto, el objetivo de este estudio es analizar la asociación de los polimorfismos $-725 C>G$ (rs1233334), -201G>A (rs1233333) y 14$\mathrm{pb}$ del/ins (rs66554220) del gen HLA-G en mujeres mexicanas con PGR.

\section{MATERIALES Y MÉTODOS}

Sujetos y extracción de ADN genómico: Para este estudio las muestras de sangre periférica se obtuvo de 114 mujeres mexicanas-mestizas atendidas en el Servicio de Obstetricia y Ginecología del Centro Médico de Occidente, Instituto Mexicano del Seguro Social del 21 de noviembre de 2011 al 28 de febrero de 2013. En el grupo de casos, se incluyeron a 58 mujeres con PGR sin anomalías cromosómicas, anatómicas uterinas y autoinmunes, en edades comprendidas entre los 18-42 años. En el grupo de controles, se incluyeron a 56 mujeres fértiles no relacionadas, en edades entre los 18-42 años con al menos 2 nacidos vivos. El Comité de Ética local aprobó la realización del estudio y todas las mujeres que participaron firmaron un consentimiento informado. El ADN genómico se extrajo de las muestras de sangre periférica utilizando el procedimiento de precipitación con sales de Miller (16).

Reacción en cadena de la polimerasa: Los 
polimorfismos $-725 \mathrm{C}>\mathrm{G}$ (rs1233334), $-201 \mathrm{G}>\mathrm{A}$ (rs1233333) y 14-pb del/ins (rs66554220) se identificaron mediante PCR-SSOP (Polymerase Chain Reaction-sequence-specific oligonucleotide probe) y PCR (Polymerase Chain Reaction), respectivamente con los iniciadores apropiados para las amplificaciones (Tabla I).

Las reacciones de PCR-SSOP y PCR se realizaron en un volumen final $20 \mu \mathrm{L}$ que incluyó 200 ng de ADN genómico, buffer de PCR IX (KCl 500 $\mathrm{mM}$, Tris-HCl $100\left(\mathrm{pH} \mathrm{9,1} \mathrm{a} 20^{\circ} \mathrm{C}\right)$ y $0,1 \%$ de Triton X-100), $2 \mathrm{mM}$ de $\mathrm{MgCl} 2,0,15 \mathrm{mM}$ de mezcla de trifosfato de desoxi-nucleótido, $2 \mathrm{mM}$ de cada iniciador y 0,05 unidades de Taq polimerasa. Las condiciones de amplificación fueron las siguientes: $94^{\circ} \mathrm{C}$ durante 5 min seguido de 30 ciclos de $94^{\circ} \mathrm{C}$ durante $30 \mathrm{~s}, 65^{\circ} \mathrm{C}$ durante $30 \mathrm{~s}\left(63^{\circ} \mathrm{C}\right.$ para $-725 \mathrm{C}>\mathrm{G}$ ), y $72^{\circ} \mathrm{C}$ durante $25 \mathrm{~s}$ con una extensión final a $72^{\circ} \mathrm{C}$ durante $7 \mathrm{~min}$. Los productos de amplificación se analizaron por electroforesis de poliacrilamida al $8 \%$ y presentaron tamaños de 127 , 203 y $210 / 224 \mathrm{pb}$ para los polimorfismos $-725 \mathrm{C}>\mathrm{G}$ (rs1233334), -201G>A (rs1233333) y 14-pb del/ins (rs66554220), respectivamente.

Análisis estadístico: Se empleó el programa http://ing.gsf.de/cgi-bin/hw/hwa1.pl para estimar el equilibrio de Hardy-Weinberg, las diferencias en las frecuencias y la asociación entre los polimorfismos y PGR, a través de la prueba de Chi-cuadrada de Pearson $\left(x^{2}\right)$ y la razón de momios (Odds Ratio), respectivamente. El desequilibrio de ligamiento se calculó con el programa Arlequin versión 3.5.1.3. Se consideró un intervalo de confianza (IC) de 95\%.

\section{RESULTADOS}

Se analizaron los polimorfismos $-725 \mathrm{C}>\mathrm{G}$ (rs1233334), -201G>A (rs1233333) y 14-pb del/ins (rs66554220) en 58 mujeres con PGR y 56 mujeres fértiles. El rango de edad en mujeres con PGR y fértiles osciló entre 31-35 años (32 \pm 5 años) y 26-30 años (30 \pm 3 años), respectivamente. Las mujeres con PGR tuvieron un número de abortos involuntarios de 2 a 6 (13 mujeres con 2, 32 con 3 , 8 con 4 , 3 con 5 y 2 con 6 abortos involuntarios). No se encontraron desviaciones significativas del equilibrio de Hardy-Weinberg en las frecuencias genotípicas de los polimorfismos $-725 \mathrm{C}>\mathrm{G}$ (rs1233334), -201G $>$ A (rs1233333) y 14-pb del/ ins (rs66554220). El genotipo -725GG se distribuyó diferente entre las mujeres con PGR $(8,6 \%)$ y las mujeres fértiles $(0 \%)$, sin embargo, no se asoció con PGR $(p=0,02601 ;$ OR $=11,484$; IC95\%=0,617213,659). El genotipo -201AA no presentó diferencia significativa en su distribución entre los grupos de estudio $(p=0,49717$; OR=0,711; IC $95 \%=0,265$ 1,908). El genotipo $14 \mathrm{pb}$ ins/ins fue discretamente más frecuente en las mujeres con PGR $(24,1 \%)$ que en las mujeres fértiles $(23,2 \%) \quad(p=0,53468$; $\mathrm{OR}=1,385$; IC95\%=0,495-3,872), diferencia no significativa (Tabla II).

Se evaluó el desequilibrio de ligamiento entre $-725 C>G$ (rs1233334) y -201G>A (rs1233333), $-725 \mathrm{C}>\mathrm{G}$ (rs1233334) y 14-pb del/ins (rs66554220), y-201G>A (rs1233333) y 14-pb del/ins (rs66554220). Los polimorfismos no se encontraron en equilibrio de ligamiento ( $D^{\prime}>0,3563 ; r^{2}<0,1140$ ) (Tabla III).

Tabla I

SECUENCIA DE INCIADORES

\begin{tabular}{ll}
\hline Iniciador & Secuencia \\
\hline HLAGF1-725C>G & GAAACTTAAGAGCTTTGTGAGACG \\
HLAGF2-725C>G & GAAACTTAAGAGCTTTGTGAGACC \\
HLAGR725 & GGGTAGCATAACCTTGGTAACC \\
HLAGF1-201G>A & CCCCACAGGCGGTGTATAGG \\
HLAGF2-201G>A & CCCCACAGGCGGTGTATAGA \\
HLAGR-201G>A & TTCGCTCACCCACCCGGACTCATT \\
HLAGEX8F14-pb del/ins & GTGATGGGCTGTTAAAGTGTCACC \\
HLAGEX8R14-pb del/ins & GGAAGGAATGCAGTTCAGCATGA
\end{tabular}


Tabla II

FRECUENCIAS GENOTÍPICAS Y ALÉLICAS Y MEDIDAS DE ASOCIACIÓN DE LOS POLIMORFISMOS CON PÉRDIDA GESTACIONAL RECURRENTE (PGR)

\begin{tabular}{|c|c|c|c|c|c|}
\hline $\begin{array}{l}\text { Polimorfismos en } \\
\text { el gen HLA-G }\end{array}$ & Genotipo & $\begin{array}{c}\text { Mujeres } \\
\text { con PGR } \\
(\%)\end{array}$ & $\begin{array}{l}\text { Mujeres } \\
\text { fértiles } \\
(\%)\end{array}$ & OR (IC95\%) & Valor $p$ \\
\hline$-725 \mathrm{C}>\mathrm{G}$ & $\begin{array}{l}\text { CC } \\
\text { CG } \\
\text { GG }\end{array}$ & $\begin{array}{r}45(77,6) \\
8(13,8) \\
5(8,6)\end{array}$ & $\begin{array}{r}47(87,5) \\
9(12,5) \\
0(0)\end{array}$ & $\begin{array}{c}- \\
0,928(0,329-2,617) \\
11,484(0,617-213,659)\end{array}$ & $\begin{array}{c}- \\
0,88824 \\
0,02601\end{array}$ \\
\hline$-201 G>A$ & $\begin{array}{l}\text { GG } \\
\text { GA } \\
\text { AA }\end{array}$ & $\begin{array}{l}19(32,8) \\
27(46,5) \\
12(20,7)\end{array}$ & $\begin{array}{l}18(32,1) \\
22(39,3) \\
16(28,6)\end{array}$ & $\begin{array}{c}- \\
1,163(0,494-2,736) \\
0,711(0,265-1,908)\end{array}$ & $\begin{array}{c}- \\
0,72990 \\
0,49717\end{array}$ \\
\hline 14-bp del/ins & $\begin{array}{l}\text { del/del } \\
\text { del/ins } \\
\text { ins/ins }\end{array}$ & $\begin{array}{l}14(24,1) \\
30(51,8) \\
14(24,1)\end{array}$ & $\begin{array}{l}18(32,1) \\
25(44,7) \\
13(23,2)\end{array}$ & $\begin{array}{c}- \\
1,543(0,642-3,709) \\
1,385(0,495-3,872)\end{array}$ & $\begin{array}{c}- \\
0,33146 \\
0,53468\end{array}$ \\
\hline Total de genotipos & & 58 & 56 & & \\
\hline $\begin{array}{l}\text { Polimorfismos en } \\
\text { el gen HLA-G }\end{array}$ & Alelo & $\begin{array}{l}\text { Mujeres } \\
\text { con PGR } \\
(\%)\end{array}$ & $\begin{array}{l}\text { Mujeres } \\
\text { fértiles } \\
(\%)\end{array}$ & OR (IC95\%) & Valor $p$ \\
\hline $\begin{array}{l}-725 C>G \\
-201 G>A\end{array}$ & $\begin{array}{l}C \\
G \\
G \\
A\end{array}$ & $\begin{array}{l}98(84,4) \\
18(15,6) \\
65(56,0) \\
51(44,0)\end{array}$ & $\begin{array}{r}103(92,0) \\
9(8,0) \\
58(51,8) \\
54(48,2)\end{array}$ & $\begin{array}{c}- \\
2,102(0,902-4,901) \\
- \\
0,843(0,500-1,419)\end{array}$ & $\begin{array}{c}- \\
0,08048 \\
- \\
0,51993\end{array}$ \\
\hline 14-bp del/ins & $\begin{array}{l}\text { del } \\
\text { ins }\end{array}$ & $\begin{array}{l}58(50,0) \\
58(50,0)\end{array}$ & $\begin{array}{l}61(54,5) \\
51(45,5)\end{array}$ & $1,196(0,711-2,013)$ & 0,49991 \\
\hline Total de alelos & & 116 & 112 & & \\
\hline
\end{tabular}

Tabla III

DESEQUILIBRIO DE LIGAMIENTO ENTRE LOS POLIMORFISMOS ANALIZADOS

\begin{tabular}{lccr}
\hline Polimorfismos del gen HLA-G & D' & $r^{2}$ & Valor p \\
\hline$-725 C>$ G y $-201 G>A$ & 0,5391 & 0,0236 & 0,1037 \\
$-725 C>$ G y 14-pb del/ins & 0,7560 & 0,0418 & 0,0306 \\
$-201 G>$ A y 14-pb del/ins & 0,3563 & 0,1140 & 0,0004 \\
\hline
\end{tabular}

\section{DISCUSIÓN}

La molécula HLA-G por tener propiedades inmunomoduladoras juega un papel primordial en la tolerancia madre-feto. Existen polimorfismos en las regiones no codificadoras del gen HLA$\mathrm{G}$ que alteran su transcripción, por tanto en este estudio se analizó la asociación de los polimorfismos -725C>G (rs1233334), -201G>A (rs1233333) y 14-pb del/ins (rs66554220) del gen HLA-G con
PGR. Los resultados revelaron que el polimorfismo -725C>G (rs1233334) sí se distribuyó de manera diferente entre las mujeres con PGR y las mujeres fértiles pero no se asoció con PGR, debido a la amplitud e inclusión de la unidad en el intervalo de confianza. En contraste, los polimorfismos -201G>A (rs1233333) y 14-pb del/ins (rs66554220) se distribuyen con similitud entre los grupos de estudio y por tanto se excluyen como factores de riego para PGR. Estudios comparables coinciden con 
lo descrito en el presente trabajo. Una investigación realizada en 58 parejas con PGR y 58 parejas fértiles no encontró asociación entre los polimorfismos $-725 \mathrm{C}>\mathrm{G}$ ( $\mathrm{rs} 1233334$ ) y 14-pb del/ins con PGR (17). Del mismo modo, otros estudios independientes no encontraron diferencias significativas en la distribución del polimorfismo 14-pb del/ins entre mujeres con PGR y mujeres fértiles $(18,19)$. Por el contrario, en la literatura se describió que el genotipo 14-pb $\mathrm{del} / \mathrm{del}$ fue más frecuente en mujeres fértiles que en mujeres con PGR ( $p=0,0053 ; O R=0,242)(20)$. Además, se ha descrito que los genotipos 14-pb ins/ins y 14-pb del/ins son más frecuentes en mujeres con $P G R$ que en mujeres fértiles y presentan un riesgo para PGR (21-23).

La búsqueda de desequilibrio de ligamiento entre los polimorfismos analizados indicó que se distribuyen de manera homogénea y que están en equilibrio de ligamiento. Es evidente que los resultados son similares a los descritos en otras poblaciones, sin embargo, existen discrepancias en la distribución de los polimorfismos debido a diferencias genéticas.

\section{CONCLUSIÓN}

Este estudio mostró que los polimorfismos en equilibrio de ligamiento $-725 \mathrm{C}>\mathrm{G}$ (rs1233334), -201G>A (rs1233333) y 14-pb del/ins (rs66554220) no se asocian con pérdida gestacional recurrente en una población mexicana.

AGRADECIMIENTOS. Al Consejo Nacional de Ciencia y Tecnología de México (CONACYT) por la beca (290733/386403) otorgada a la Dra. Angela Porras Dorantes.

\section{REFERENCIAS}

1. Ford HB, Schust DJ. Recurrent pregnancy loss: etiology, diagnosis, and therapy. Rev Obstet Gynecol 2009;2(2):76-83.

2. Bashiri A, Ratzon R, Amar S, Serjienko R, Mazor M, Shoham-Vardi I. Two vs. three or more primary recurrent pregnancy losses--are there any differences in epidemiologic characteristics and index pregnancy outcome? J Perinat Med 2012;40(4):365-71.

3. Shapira E, Ratzon R, Shoham-Vardi I, Serjienko R, Mazor M, Bashiri A. Primary vs. secondary recurrent pregnancy loss--epidemiological characteristics, etiology, and next pregnancy outcome. J Perinat Med 2012;40(4):389-96.

4. Chakraborty P, Goswami SK, Rajani S, Sharma S, Kabir SN, Chakravarty B, et al. Recurrent pregnancy loss in polycystic ovary syndrome: role of hyperhomocysteinemia and insulin resistance. PLoS One 2013;8(5):e64446.
5. Aruna M, Sudheer PS, Andal S, Tarakeswari S, Reddy AG, Thangaraj K, et al. HLA-G polymorphism patterns show lack of detectable association with recurrent spontaneous abortion. Tissue Antigens 2010;76(3):216-22.

6. Curigliano G, Criscitiello C, Gelao L, Goldhirsch A. Molecular pathways: human leukocyte antigen $G$ (HLA-G). Clin Cancer Res 2013;19(20):5564-71.

7. Yan WH, Fan LA, Yang JQ, Xu LD, Ge Y, Yao FJ. HLA-G polymorphism in a Chinese Han population with recurrent spontaneous abortion. Int $\mathrm{J}$ Immunogenet 2006;33(1):55-8.

8. Donadi EA, Castelli EC, Arnaiz-Villena A, Roger M, Rey $D$, Moreau $P$. Implications of the polymorphism of HLA-G on its function, regulation, evolution and disease association. Cell Mol Life Sci 2011;68(3):369-95.

9. Aldrich $C L$, Stephenson MD, Karrison T, Odem RR, Branch DW, Scott JR, et al. HLA-G genotypes and pregnancy outcome in couples with unexplained recurrent miscarriage. Mol Hum Reprod 2001;7(12):116772.

10. Ober C, Aldrich CL, Chervoneva I, Billstrand C, Rahimov F, Gray HL, et al. Variation in the HLA-G promoter region influences. Am J Hum Genet 2003;72(6):142535.

11. Hviid TV. HLA-G in human reproduction: aspects of genetics, function and pregnancy complications. Hum Reprod Update 2006;12(3):209-32.

12. Ober C, Billstrand C, Kuldanek S, Tan Z. The miscarriage-associated HLA-G-725G allele influences transcription rates in JEG-3 cells. Hum Reprod 2006;21(7):1743-8.

13. Mendes-Junior CT, Castelli EC, Simões RT, Simões AL, Donadi EA. HLA-G 14-bp polymorphism at exon 8 in Amerindian populations from the Brazilian Amazon. Tissue Antigens 2007;69(3):255-60.

14. Gonzalez A, Alegre E, Torres MI, Díaz-Lagares A, Lorite $\mathrm{P}$, Palomeque $\mathrm{T}$, et al. Evaluation of HLA-G5 plasmatic levels during pregnancy and relationship with the 14-bp polymorphism. Am J Reprod Immunol 2010;64(5):367-74.

15. Tian W, Cai JH, Wang F, Li LX, Cao Y. HLA-G*0105N and HLA-G 14 bp dimorphisms in exon 8 in four distinct populations in mainland China. Tissue Antigens 2010;75(3):227-34.

16. Miller, S, Dykes D, Polesky H. A simple salting out procedure for extracting DNA from human nucleated cells. Nucleic Acids Res 1988;16(3):1215.

17. Sipak-Szmigiel O, Cybulski C, Lubiński J, RoninWalknowska E. HLA-G polymorphism in a Polish population and reproductive failure. Tissue Antigens 2008;71(1):67-71.

18. Hviid TV, Hylenius S, Hoegh AM, Kruse C, Christiansen OB. HLA-G polymorphisms in couples with recurrent spontaneous abortions. Tissue Antigens 2002;60(2):122-32.

19. Yan WH, Lin A, Chen XJ, Dai MZ, Gan LH, Zhou $\mathrm{MY}$, et al. Association of the maternal 14-bp insertion polymorphism in the HLA-G gene in women with recurrent spontaneous abortions. Tissue Antigens 2006;68(6):521-3.

20. Shankarkumar U, Shankarkumar A, Chedda Z, Ghosh 
K. Role of $14-b p$ deletion/insertion polymorphism in exon 8 of the HLA-G gene in recurrent spontaneous abortion patients. J Hum Reprod Sci 2011;4(3):143-6.

21. Zhu Y, Huo Z, Lai J, Li S, Jiao H, Dang J, Jin C. Case-control study of a HLA-G 14-bp insertion-deletion polymorphism in women with recurrent miscarriages. Scand J Immunol 2010;71(1):52-4.
22. Xue S, Yang J, Yao F, Xu L, Fan L. Recurrent spontaneous abortions patients have more $-14 \mathrm{bp} /+14$ bp heterozygotes in the 3'UT region of the HLA-G gene in a Chinese Han population. Tissue Antigens 2007;69(1):153-5

23. Tripathi $P$, Abbas A, Naik S, Agrawal S. Role of 14bp deletion in the HLA-G gene in the maintenance of pregnancy. Tissue Antigens 2004;64(6):706-10. 